Int. J. Morphol.,

32(3):860-865, 2014

\title{
Trígono Cistohepático: Área Crítica Anatómica para la Seguridad Quirúrgica
}

\author{
Trigonum Cystohepaticum: Critical Anatomical Area for Surgical Safety
}

\author{
Rubén Daniel Algieri*; María Soledad Ferrante**; Juan Sebastián Ugartemendía***; \\ María de las Mercedes Bernadou****; Lucas Pina ${ }^{* * * * *}$ \& Agustín Álvarez Escalante ${ }^{* * * * *}$
}

\begin{abstract}
ALgieri, R. D.; FERRANTE, M. S; UGARTEMENDÍA, J. S.; BERNADOU, M. M.; PINA, L. \& ÁLVAREZ, E. A. Trígono cistohepático: área crítica anatómica para la seguridad quirúrgica. Int. J. Morphol., 32(3):860-865, 2014.

RESUMEN: El conocimiento de la anatomía de la vía biliar y sus variantes para la realización de una cirugía segura, resulta fundamental. La extirpación de la vesícula requiere cuidadosa atención, conocer muy bien la anatomía de la región, teniendo en cuenta la posibilidad de variaciones anatómicas. La mala interpretación de la anatomía percibida más que una falta en la destreza técnica es la causa de la lesión de la vía biliar durante la colecistectomía. Diferenciar el límite y el contenido del trígono cistohepático. Diseñar las áreas de Visión Crítica y de Seguridad como medida de seguridad en el paciente quirúrgico. Revisión de 458 partes quirúrgicos de colecistectomías de enero/2010 a octubre/2012, en el Servicio de Cirugía General del Hospital Aeronáutico Central, y disección de 12 cadáveres adultos formolizados al 10\% en la III Cátedra de Anatomía - Facultad de Medicina - Universidad de Buenos Aires. De 458 colecistectomías, se clasificaron los partes quirúrgicos, dividiéndose según menciona: triángulo de Calot en 247 (53,93\%); triángulo hepatocístico en 59 (12,88\%); área de visión crítica en 152 (33,18\%); ninguno mencionó al triángulo de Budde o trígono cistohepático. Se disecaron 12 cadáveres adultos donde se identificó: arteria cística originándose de arteria hepática derecha en 9 (75\%); originándose de arteria hepática izquierda en 2 (16,66\%) y originándose de arteria hepática en 1 (8,34\%). En 7 (58,35\%) se la visualiza en trígono cistohepático. El conocimiento de la anatomía de la vía biliar y sus variantes para la realización de una cirugía segura, resulta fundamental. El triángulo descrito por Calot corresponde a la mitad inferior del triángulo descrito por Buddé. El sector lateral (Triangulo de Seguridad) es el verdadero área de visión critica a disecar por la menor probabilidad de lesionar estructuras nobles.
\end{abstract}

PALABRAS CLAVE: Trígono cistohepático; Triángulo hepatocístico; Triángulo de Calot; Triángulo de Buddé.

\section{INTRODUCCIÓN}

El conocimiento de la anatomía de la vía biliar y sus variantes para la realización de una cirugía segura, resulta fundamental. La extirpación de la vesícula, sea laparoscópica o convencional; es una operación que requiere cuidadosa atención, conocer muy bien la anatomía de la región, teniendo en cuenta la posibilidad de encontrar anomalías o variaciones anatómicas (McAneny, 2008; Cachoeira et al., 2012). La mala interpretación de la anatomía percibida más que una falta en la destreza técnica, es la causa de la lesión de la vía biliar durante la colecistectomía. Hay diversas estrategias de disección quirúrgica para minimizar el riesgo de lesión de la vía biliar, la más confiable es la de "visión crítica" (critical view) (Vettoretto et al., 2011) descrita por
Strasberg (2005a). Para alcanzar la visión crítica, el trígono cistohepático pone en evidencia al conducto cístico, arteria cística, vena cística y la base del lecho hepático que son expuestos (Strasberg, 2005b). Hoy, continúan existiendo discrepancias en el reconocimiento anatómico estipulado por las técnicas quirúrgicas vigentes (Strasberg, 2002, 2005a, 2005b; Strasberg \& Brunt, 2010; Vandamme et al., 1969), así que las áreas descriptas por Calot y Budde han sido punto de análisis de varios autores (Abdalla et al., 2013; Balija et al., 1999; Loukas et al., 2008). El objetivo de este trabajo es diferenciar el límite y el contenido del trígono cistohepático; y diseñar las áreas de Visión Crítica y de Seguridad como medida de seguridad en el pacientequirúrgico.

* Médico, UBA. Especialista en Cirugía General. Prof. Adjunto de Anatomía e Histología, UM. Prof. Adjunto de Anatomía, Facultad de Medicina, UBA. Director del Laboratorio de Esplacnología (LE), Centro de Investigaciones en Anatomía Aplicada (CIAA), III Cátedra de Anatomía, Facultad de Medicina, Universidad de Buenos Aires, Buenos Aires, Argentina.

** Médica, UBA. Especialista en Cirugía General. Docente Adscripta de Anatomía e Histología, UM. Instructora de Residentes de Cirugía General del Hospital Aeronáutico Central, Buenos Aires, Argentina.

*** Médico, UBA. Residente de Cirugía General del Hospital Aeronáutico Central, Buenos Aires, Argentina.

${ }^{* * * * *}$ Médica, UBA. Jefe de Trabajos Prácticos, III Cátedra de Anatomía, Facultad de Medicina Universidad de Buenos Aires, Buenos Aires, Argentina.

******Ayudante de Anatomía, III Cátedra de Anatomía, Facultad de Medicina Universidad de Buenos Aires, Buenos Aires, Argentina. 


\section{MATERIAL Y MÉTODO}

Se realizó un estudio descriptivo, con la revisión de 458 partes quirúrgicos de colecistectomías comprendidas en el período enero/2010 a octubre/2012, en el Servicio de Cirugía General del Hospital Aeronáutico Central (Figs. 1-3), y la disección de 12 cadáveres adultos (5 hombres y 7 mujeres) formolizados al $10 \%$ en la III Cátedra de Anatomía de la Facultad de Medicina de la Universidad de Buenos Aires (Figs. 4-10).

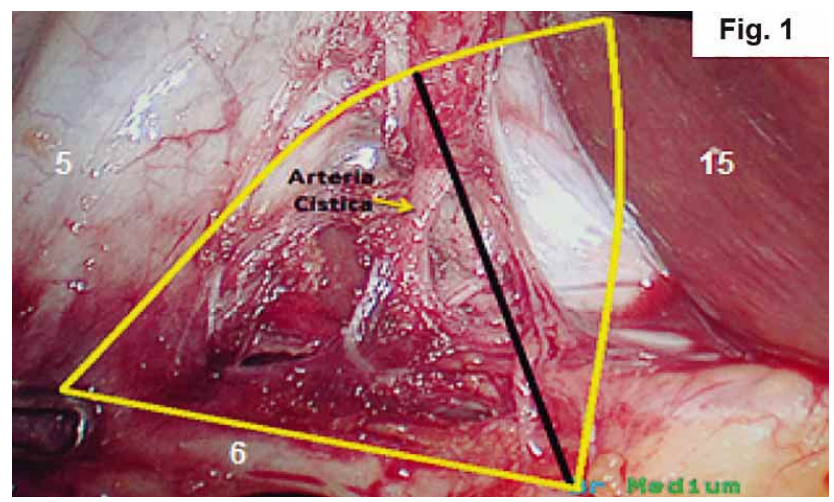

Fig. 1. Disección del triángulo de Budde en cirugía laparoscópica. 5) Vesícula biliar; 6) Conducto cístico; 15) Hígado.

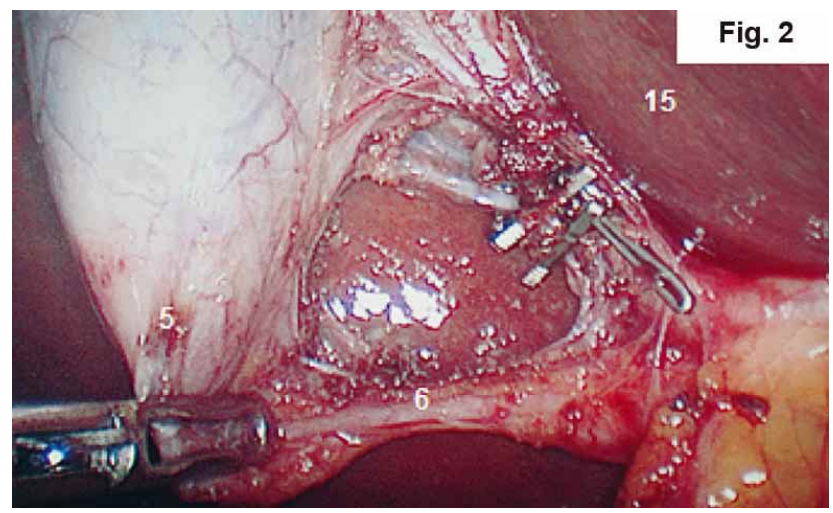

Fig. 2. Disección completa del Triángulo de Seguridad. 5) Vesícula biliar; 6) Conducto cístico; 15) Hígado.

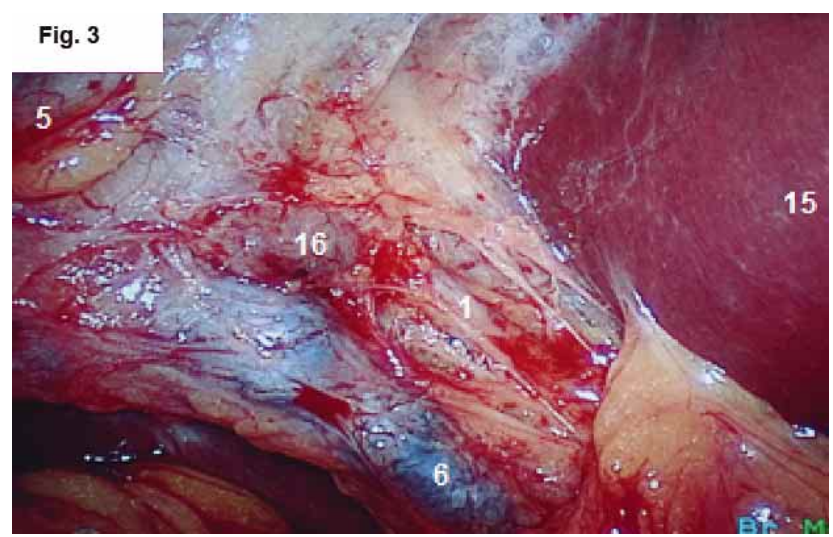

Fig. 3. Nodo linfático (Mascagni). 5) Vesícula biliar; 6) Conducto cístico; 15) Hígado.

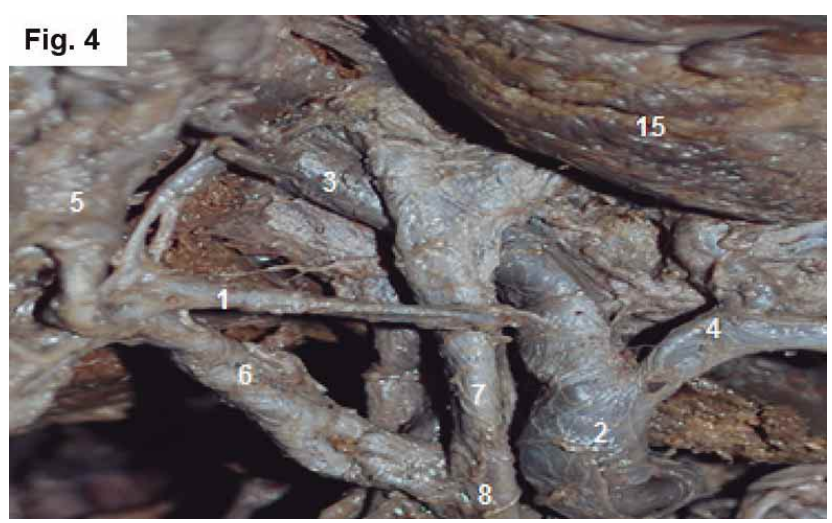

Fig. 4. Arteria cística naciendo a la izquierda del conducto hepático. 1) Arteria cística; 2) Arteria hepática; 3) Rama derecha de la arteria hepática; 4) Rama izquierda de la arteria hepática; 5) Vesícula biliar; 6) Conducto cístico; 7) Conducto hepático; 8) Conducto colédoco; 15) Hígado.

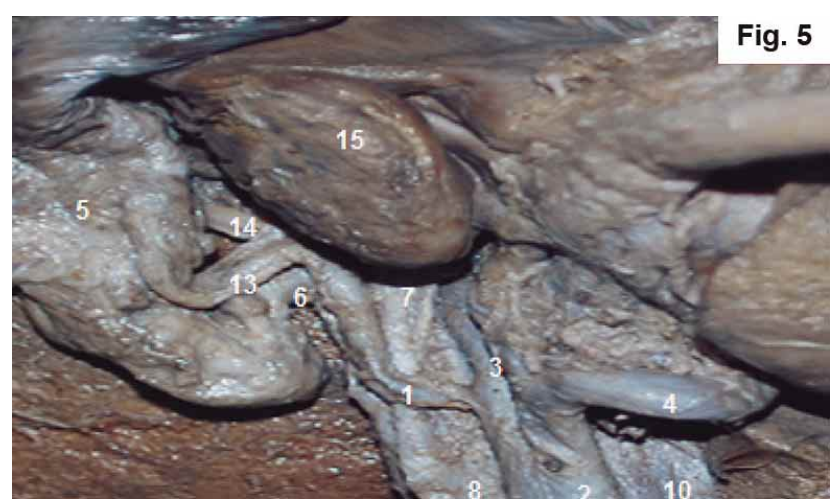

Fig. 5. Rama derecha de la arteria hepática y arteria cística por delante del conducto colédoco. 1) Arteria cística; 2) Arteria hepática; 3) Rama derecha de la arteria hepática; 4) Rama izquierda de la arteria hepática; 5) Vesícula biliar; 6) Conducto cístico; 7) Conducto hepático; 8) Conducto colédoco; 10) Vena porta; 13) Rama anterior de la arteria cística; 14) Rama posterior de la arteria cística; 15) Hígado.

\section{RESULTADOS}

De las 458 colecistectomías analizadas, 437 $(95,41 \%)$ se realizaron por vía laparoscópica (de las cuales $22(5,03 \%)$ se convirtieron) y $21(4,59 \%)$ fueron convencionales. Se clasificaron los partes quirúrgicos, dividiéndose los mismos según menciona como: triángulo de Calot en 247 (53,93\%), triángulo hepatocístico en 59 $(12,88 \%)$ y área de visión crítica en $152(33,18 \%)$; ninguno hizo referencia al triángulo de Budde y o trígono cistohepático.

En los cadáveres disecados, se identificó la arteria cística originándose de la arteria hepática derecha en 9 


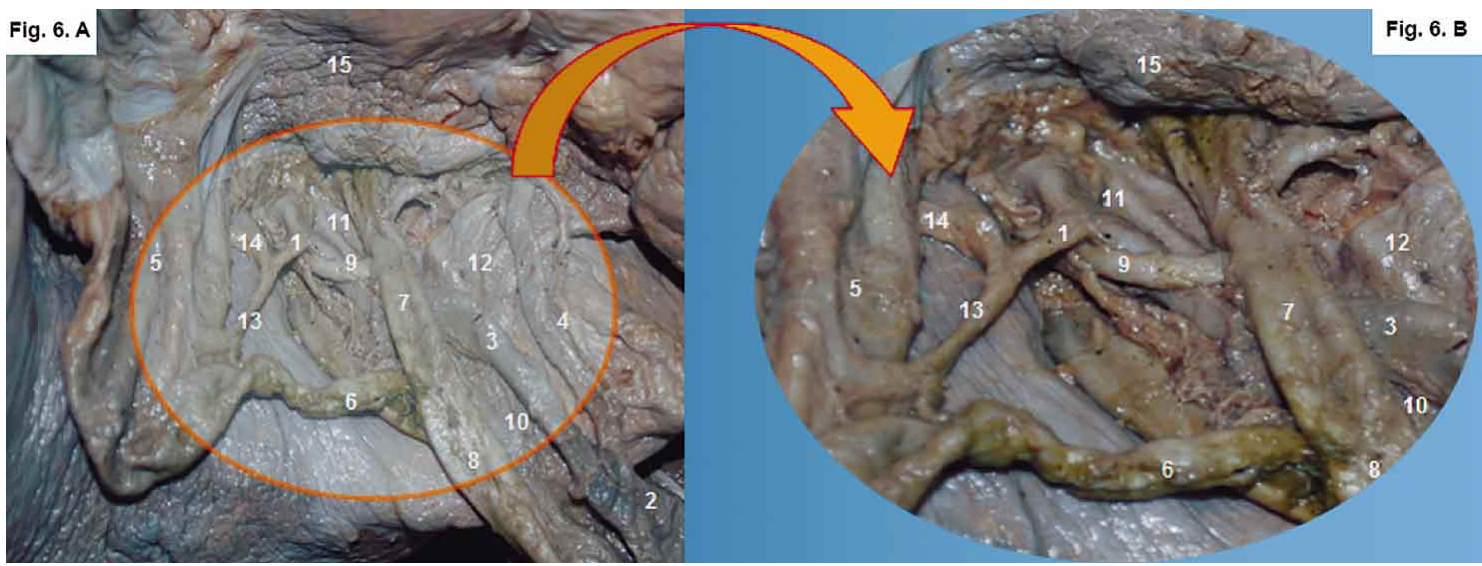

Fig. 6. A. Arteria cística con sus ramas anterior y posterior y conducto biliar anómalo en el triangulo de Budde. B. Rama derecha de la arteria hepática y rama derecha de la vena porta en el sector medial del triángulo de Budde. 1) Arteria cística; 3) Rama derecha de la arteria hepática; 5) Vesícula biliar; 6) Conducto cístico; 7) Conducto hepático; 8) Conducto colédoco; 9) Conducto biliar anómalo; 10) Vena porta; 11) Rama derecha de la vena porta; 12) Rama Izquierda de la vena porta; 13) Rama anterior de la arteria cística; 14) Rama posterior de la arteria cística; 15) Hígado.

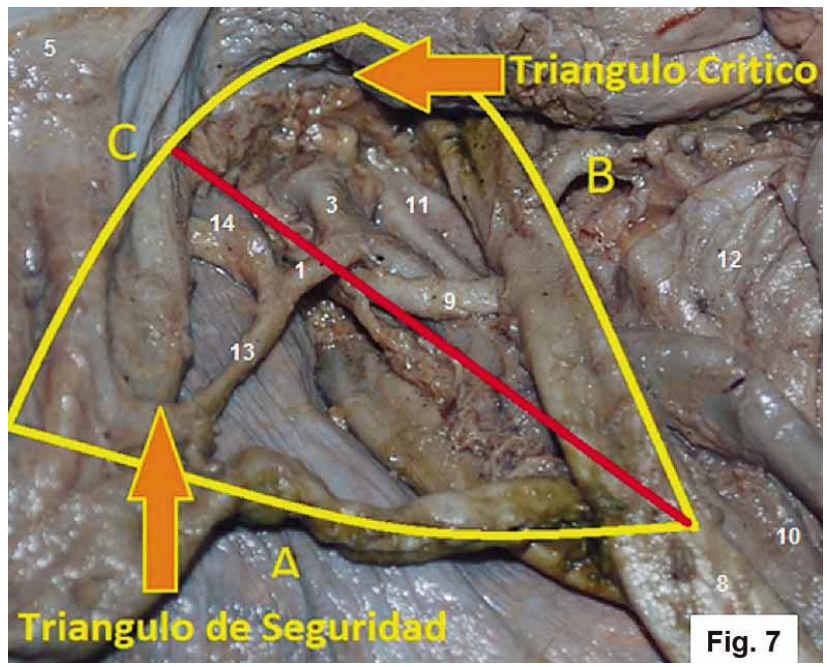

Fig. 7. Disección del triangulo de Budde. División esquemática de dicho triángulo en uno medial (Critico) y otro lateral (de Seguridad). A: Conducto Cístico; B: Conducto Hepático; C: Hígado. 1) Arteria cística; 3) Rama derecha de la arteria hepática; 9) Conducto biliar anómalo; 10) Vena porta; 11) Rama derecha de la vena porta; 12) Rama Izquierda de la vena porta; 13) Rama anterior de la arteria cística; 14) Rama posterior de la arteria cística.

Fig. 9. Ramas anterior y posterior de la arteria cística en el Triángulo de Seguridad. 3) Rama derecha de la arteria hepática; 9) Conducto biliar anómalo; 11) Rama derecha de la vena porta; 13) Rama anterior de la arteria cística; 14) Rama posterior de la arteria cística.

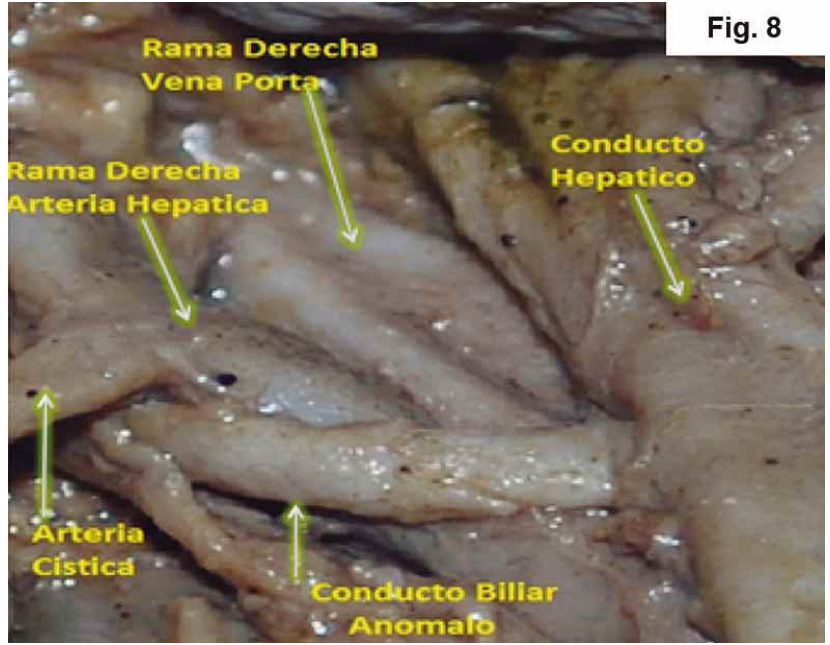

Fig. 8. Triángulo Crítico.

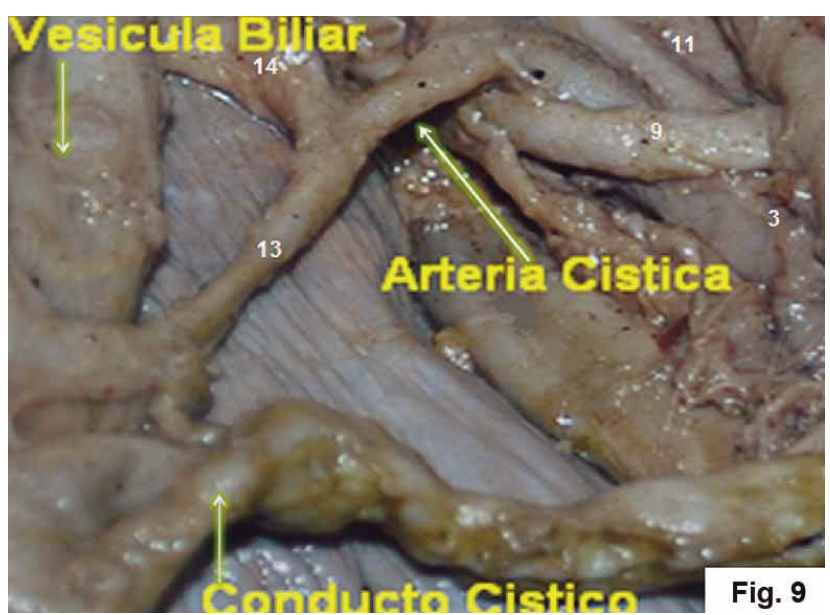




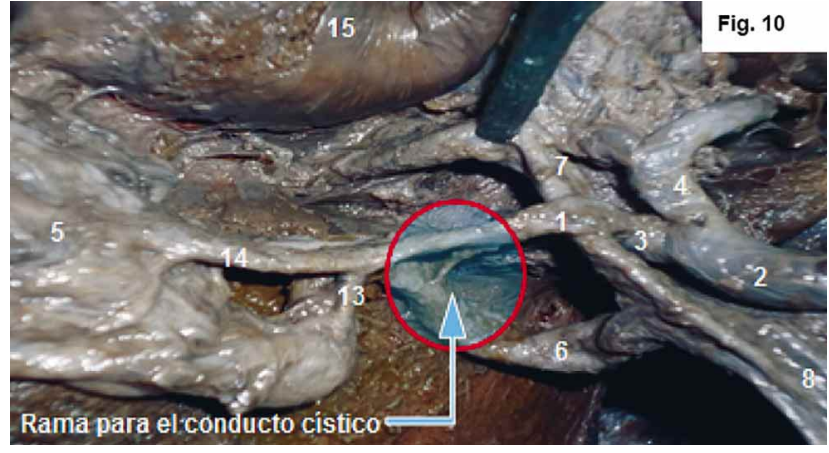

Fig. 10. Arteria cística, sus ramas terminales y colateral para el conducto cístico.

(75\%) casos, en la izquierda en $2(16,66 \%)$ y desde la arteria hepática en $1(8,34 \%)$; todas ellas con sus ramas anterior y posterior $(100 \%)$. Se identificaron arterias císticas dobles en $2(16,66 \%)$ disecciones. En $7(58,35 \%)$ casos, se la visualizó en el trígono cistohepático (triángulo hepatocístico), en el extremo lateral próximo a la vesícula. En otros casos no se encontraban contenidas en el triángulo mencionado, siendo $1(8,33 \%)$ posterior al conducto hepático común, $1(8,33 \%)$ anterior al mismo conducto, 1 $(8,33 \%)$ posterior al conducto colédoco y $1(8,33 \%)$ anterior a éste. En $1(8,33 \%)$ no se relacionaba con ese sector de la vía biliar. Se observó en $2(16,66 \%)$ casos, pequeñas venas que penetran en el hígado y desembocan directamente en ramas intrahepáticas de la vena porta. En los 10 $(83,34 \%)$ restantes, una vena cística desembocó en la rama derecha de la vena porta.

\section{DISCUSIÓN}

Jean François Calot (1861-1944), nació en Arrens, Francia, y estudió en la Universidad de París, su tesis doctoral la llamó "De la colecystectomie" y estuvo terminada en 1890 (de la Garza-Villaseñor, 2001) (Fig. 11); en ella describe un triángulo isósceles dado por la arteria y conducto cístico en sus límites superior e inferior respectivamente, y por el conducto hepático medialmente (Fig. 12). Refiere que la arteria hepática derecha puede contribuir en un tercio de los casos con su límite superior (Calot, 1890). La terminología anatómica internacional contempla la existencia de éste triángulo bajo el término "trígono cistohepático" (FCAT, 2001). Calot insistió en que "el cirujano debe trabajar por vista y no por fe" (Calot). Budde (Fig. 13) describe en 1906 un triángulo anatómico comprendido entre el conducto cístico, el conducto hepático y el hígado (Fig. 1), y lo denominó "gallengangsdreieck"; interpretado como "triángulo del conducto biliar", conte- niendo al pedículo cístico (arteria cística, vena cística y ramilletes del plexo celíaco), la arteria hepática derecha, la rama derecha de la vena porta (Fig. 6B), conductos biliares anómalos (Fig. 6A) y nodos linfáticos (Fig. 14

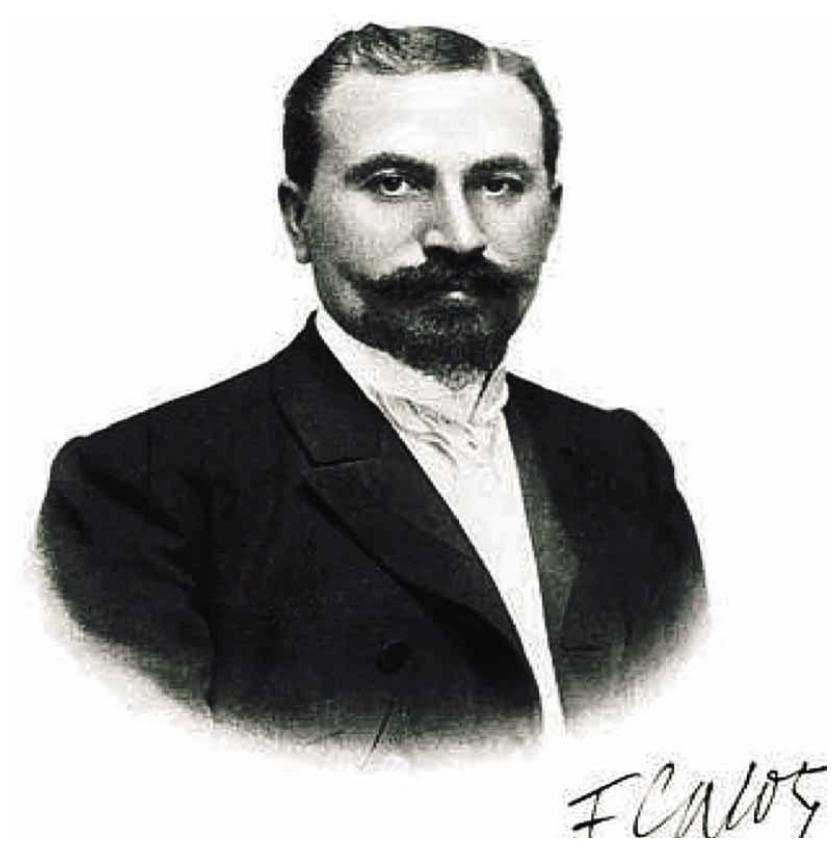

Fig. 11. Jean Francois Calot (1861-1944).

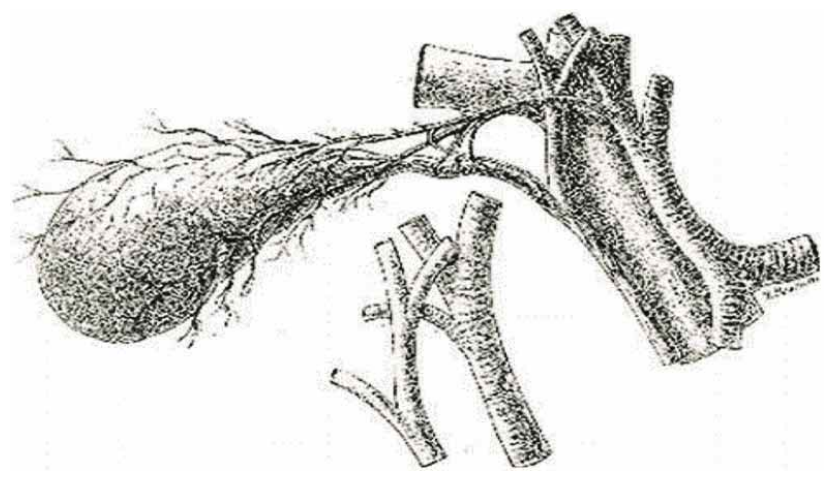

Fig. 12. Tesis doctoral de Calot (1890) "De la cholecystectomie".

II.

Beiträge zur Kenntnis der Topographio der normalen A. hepatica und ihrer Varietäten sowie der Blatversorgung der Leber.

Von

Dr. Budde in Bocholt i. W.

(Mit 22 Abbildungen.)

Fig. 13. Título del trabajo original de Budde (1906). 


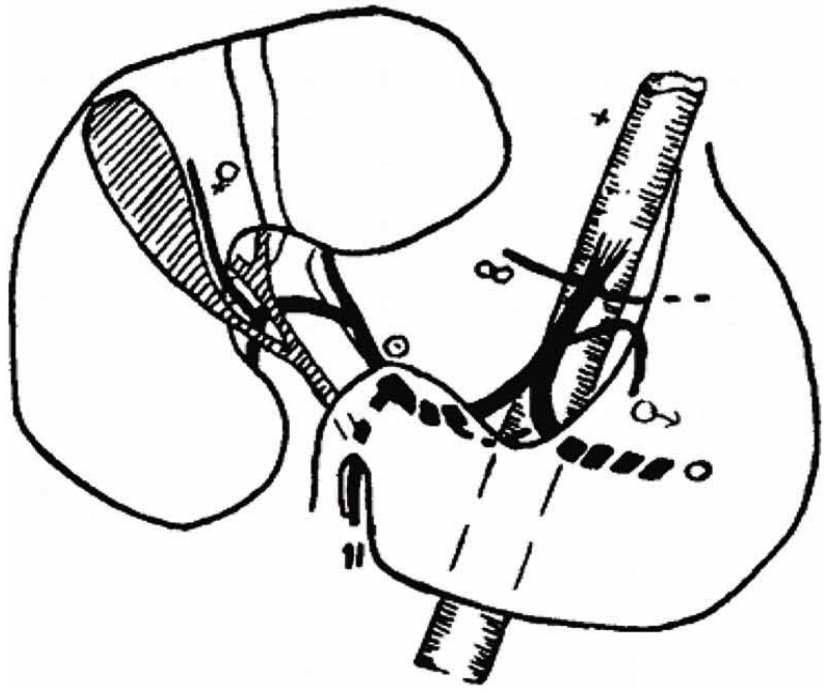

Fig. 14. Esquema de la arteria hepática y sus ramas (Budde, 1906).

(Budde), Fig. 3). Se puede dividir al triángulo descrito por Budde en dos sectores (Fig. 7), uno medial (Triángulo Crítico) (Fig. 8) y otro lateral (Triángulo de Seguridad) (Figs. 2 y 9), siendo su limite de separación una línea que va desde el ángulo hepatocístico, pasando por el nacimiento de la arteria cística y alcanzando el margen hepático (Rouviere \& Delmas, 2005).

El desconocimiento de las descripciones originales, el término acuñado por la terminología anatómica internacional o la interpretación de la traducción de "gallangangsdreick", realizado a lo largo de los años, llevó a errores y confusión en el reconocimiento de los límites de estas áreas topográficas.

\section{CONCLUSIÓN}

El conocimiento de la anatomía de la vía biliar y sus variantes para la realización de una cirugía segura, resulta fundamental. El triángulo descrito por Calot corresponde a la mitad inferior del triángulo descrito por Buddé. Cuando se tracciona la vesícula hacia afuera y a la derecha, aumenta la abertura del ángulo entre conductos hepático y cístico. En ninguno de nuestros casos se mencionó en el parte quirúrgico al triángulo descrito por Budde. Se debe tener en cuenta que el sector lateral (Triangulo de Seguridad) es el verdadero área de visión critica a disecar por los cirujanos, ya que en este sector existe menor probabilidad de lesionar estructuras nobles (rama derecha de la arteria hepática, rama derecha de la vena porta, conductos biliares anómalos), y produciendo una mayor seguridad en la técnica quirúrgica. Por lo que es de suma importancia reconocer anatómicamente estos triángulos a la hora de realizar ligadura de arteria cística durante la colecistectomía. Cualquiera sea el origen de esta arteria, siempre se debe explorar en el sector lateral del triángulo descrito por Budde, donde su presencia es constante; y su disección y exploración genera menor riesgo de lesión de elementos nobles que ponen en riesgo la salud del enfermo.

ALGIERI, R. D.; FERRANTE, M. S; UGARTEMENDÍA, J. S.; BERNADOU, M. M.; PINA, L. \& ÁLVAREZ, E. A. Trigonum cystohepaticum: critical anatomical area for surgical safety. Int. J. Morphol., 32(3):860-865, 2014.

SUMMARY: Knowing the anatomy of the bile duct and its anatomical variations becomes essential to safely perform any surgery. Gallbladder resection requires careful attention: knowing the region's anatomy by heart and taking into account the possibility of anatomical variations. Misunderstanding the anatomy is not only a failure in technical ability but also a cause of injury to the bile duct during a cholescystectomy. The objectives of this study were, to distinguish the boundaries and content of the trigonum cystohepaticum. Furthermore, to design the areas of Safety and Critical Vision as a safety measure for the patient undergoing surgery. Analysis of 458 surgical reports on cholecystectomies performed from January 2010 to October 2012 by the Hospital Aeronáutico's General Surgery Department, and dissection of 12 adult cadavers preserved in a 10\% formalin solution at the IIIrd Chair of Anatomy, School of Medicine, University of Buenos Aires. From 458 cholecystectomies, surgical reports were classified as mentioning: Calot triangle, 247 (53.93\%); cystohepatic triangle, 59 (12.88\%); critical vision area, $152(33.18 \%)$. None of them mentioned Buddé triangle or trigonum cystohepaticum. Twelve adult cadavers were dissected in which we identified the cystic artery: originating from right hepatic artery, 9 (75\%); originating from left hepatic artery, 2 (16.66\%); and originating from hepatic artery, 1 (8.34\%). Trigonum cystohepaticum is observed in 7 cadavers (58.35\%). Knowing the anatomy of the bile duct and its anatomical variations becomes essential to safely perform any surgery. The triangle described by Calot is the lower half of the triangle described by Buddé. The lateral portion (Safety Triangle) is the area of critical vision to be dissected due to the lower probability of injuring noble structures.

KEY WORDS: Trigonum cystohepaticum; Cystohepatic triangle; Calot triangle; Buddé triangle. 


\section{REFERENCIAS BIBLIOGRÁFICAS}

McAneny, D. Open cholecystectomy. Surg. Clin. North Am., 88(6):1273-94, 2008.

Strasberg, S. M. Avoidance of biliary injury during laparoscopic cholecystectomy. J. Hepatobiliary Pancreat. Surg., 9(5):5437, 2002.

Strasberg, S. M. Biliary injury in laparoscopic surgery: part 1. Processes used in determination of standard of care in misidentification injuries. J. Am. Coll. Surg., 201(4):598-603, 2005a.

Strasberg, S. M. Biliary injury in laparoscopic surgery: part 2. Changing the culture of cholecystectomy. J. Am. Coll. Surg., 201(4):604-11, $2005 b$.

Strasberg, S. M. \& Brunt, L.M. Rationale and use of the critical view of safety in laparoscopic cholecystectomy. J. Am. Coll. Surg., 211(1):132-8, 2010.

Rouviere, H. \& Delmas, A. Anatomía Humana, Descriptiva, Topográfica y Funcional. 11 ${ }^{\mathrm{a}}$ ed. Barcelona, Elsevier, 2005.

Vandamme, J. P.; Bonte, J. \& Van der Schueren, G. A revaluation of hepatic and cystic arteries. The importance of the aberrant hepatic branches. Acta Anat. (Basel), 73(2):192-209, 1969.

Vettoretto, N.; Saronni, C.; Harbi, A.; Balestra, L.; Taglietti, L. \& Giovanetti, M. Critical view of safety during laparoscopic cholecystectomy. JSLS, 15(3):322-5, 2011.

\author{
Dirección para Correspondencia: \\ Rubén Daniel Algieri \\ Médico, Especialista en Cirugía General \\ III Cátedra de Anatomía, Facultad de Medicina \\ Universidad de Buenos Aires \\ Paraguay 2155 \\ Ciudad Autónoma de Buenos Aires \\ ARGENTINA \\ Email: rdalgieri08@hotmail.com
}

Recibido : 04-05-2014

Aceptado: 26-05-2014 